\title{
A Patient With Delayed Development Resulting From De Novo Duplication of 7q36.1-q36.3 and Deletion of 9p24.3
}

\author{
Asayeon Choi, $\mathrm{MD}^{1}$, Ja-Young $\mathrm{Oh}, \mathrm{MD}^{1}$, Myungshin $\mathrm{Kim}, \mathrm{MD}, \mathrm{PhD}^{2,3}$, \\ Woori Jang, $\mathrm{MD}^{2,3}$, Dae-Hyun Jang, $\mathrm{MD}, \mathrm{PhD}^{1}$
}

\begin{abstract}
${ }^{1}$ Department of Rehabilitation Medicine, Incheon St. Mary's Hospital, College of Medicine, The Catholic University of Korea, Incheon; ${ }^{2}$ Department of Laboratory Medicine, College of Medicine, The Catholic University of Korea, Seoul;

${ }^{3}$ Catholic Genetic Laboratory Center, College of Medicine, The Catholic University of Korea, Seoul, Korea
\end{abstract}

\begin{abstract}
Patients with a duplication from $7 \mathrm{q} 36$ to the terminus or a deletion of $9 \mathrm{p} 24$ have been reported, whereas those harboring both mutations have not. Here, we report a patient with simultaneous de novo 7q36.1-q36.3 duplication and 9p24.3 deletion. A 6-year-old boy presented with speech developmental delay, microcephaly, and dysmorphic features, including a long face and small nose. Chromosome and array comparative genomic hybridization analyses revealed 46,XY,dup(7)(q36.1-q36.3) and $\operatorname{del}(9)(\mathrm{p} 24.3)$. The sizes of the duplication and deletion were $9.9 \mathrm{Mb}$ and $1.9 \mathrm{Mb}$, respectively. The duplication of chromosome 7 contained 68 known genes, of which 3 are related with entries in the Developmental Disorders Genotype-to-Phenotype (DDG2P) database. The deletion of chromosome 9 contained 6 known genes, of which 2 are in the DDG2P database. We investigated the genotype and phenotype in this patient, and reviewed the relevant literatures for possible clinical presentation in these variations.
\end{abstract}

Keywords Developmental disabilities, Chromosome disorders

\section{INTRODUCTION}

There have been cases reporting either duplication of $7 q 36$ to the terminus or deletion of 9p24; however none with both simultaneously. Here, we report a patient with simultaneous de novo 7q36.1-q36.3 duplication and 9p24.3 deletion. A 6-year-old boy was referred for the evaluation of developmental delay. He had microcephaly and mild dysmorphic features, such as a long face and small nose. Chromosome and array comparative genomic hybridization analyses revealed 46,XY, dup(7)(q36.1q36.3) and $\operatorname{del}(9)(\mathrm{p} 24.3)$. The sizes of the duplication

Received October 6, 2016; Accepted October 24, 2016

Corresponding author: Dae-Hyun Jang

Department of Rehabilitation Medicine, Incheon St Mary's Hospital, College of Medicine, The Catholic University of Korea, 56 Dongsu-ro, Bupyeonggu, Incheon 21431, Korea. Tel: +82-32-280-5207, Fax: +82-32-280-5040, E-mail: dhjangmd@naver.com

ORCID: Asayeon Choi (http://orcid.org/0000-0002-4666-5185); Ja-Young Oh (http://orcid.org/0000-0002-0230-7677); Myungshin Kim (http://orcid. org/0000-0001-8632-0168); Woori Jang (http://orcid.org/0000-0001-8376-0133); Dae-Hyun Jang (http://orcid.org/0000-0001-8293-084X).

(c) This is an open-access article distributed under the terms of the Creative Commons Attribution Non-Commercial License (http://creativecommons.org/ licenses/by-nc/4.0) which permits unrestricted noncommercial use, distribution, and reproduction in any medium, provided the original work is properly cited. Copyright (c) 2017 by Korean Academy of Rehabilitation Medicine 
and deletion were $9.9 \mathrm{Mb}$ and $1.9 \mathrm{Mb}$, respectively. The duplication of chromosome 7 contained 68 known genes, 3 of which are related to entries in the Developmental Disorders Genotype-to-Phenotype (DDG2P) database. The deletion of chromosome 9 contained 6 known genes, 2 of which are in the DDG2P database. This study investigated the genotype and phenotype in this patient, and reviewed the relevant literatures for possible clinical presentations in these variations.

\section{CASE REPORT}

A 6-year-old boy was transferred to our institution from a local hospital for evaluation for developmental delays in speech and language. He could communicate using about 10 words, but could not form sentences. He could not read or write, and was only able to draw circles. The patient showed some growth delay as well; his weight was $17.8 \mathrm{~kg}$ (<3rd percentile), height $115.8 \mathrm{~cm}$ (10th-15th percentile), and head circumference $48.5 \mathrm{~cm}$ ( $<3 \mathrm{rd}$ percentile). He was born preterm at 35 weeks of gestational age; however there was no other prepartum or postpartum event. His birth weight was 2,400 g ( $<3$ rd percentile). When he was 2 years old, operations for both inferior oblique muscle over-action with exotropia were performed. Until the age of 3 years, he had been hospitalized several times due to recurrent episodes of pneumonia.
He started to walk with hand support at 23 months old, and independent outdoor gait was possible when he was 36 months old. He received speech and sensory integration therapy at the private center, and was enrolled in kindergarten for disabled children 3 years ago. His parents were healthy, and there was no remarkable finding in his pedigree. His siblings (one sister and one brother)
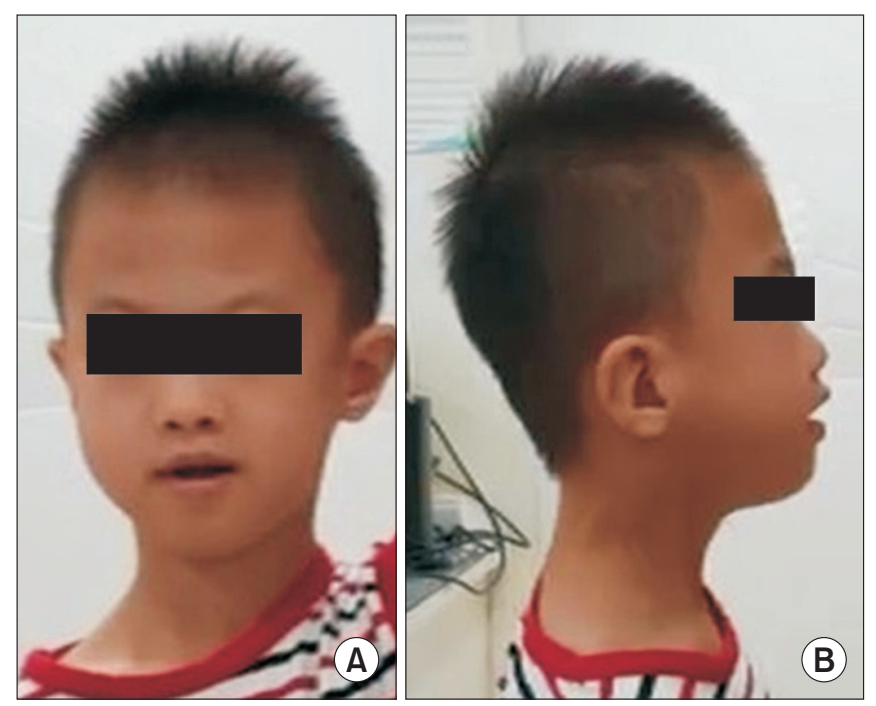

Fig. 1. Antero-posterior view (A) and lateral view (B) of the patient showed microcephaly and mild facial dysmorphic features, such as a long face with high forehead, small nose and ears.
A

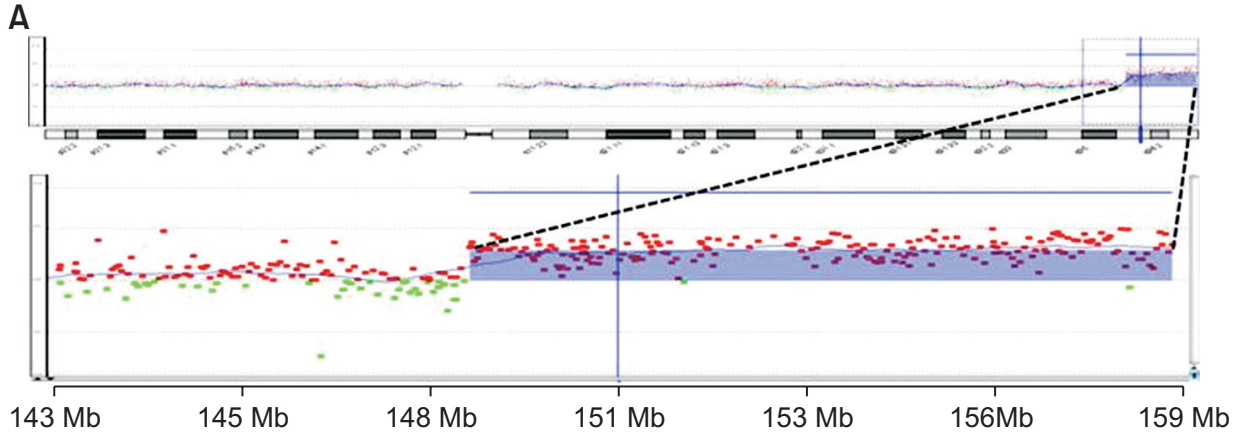

B

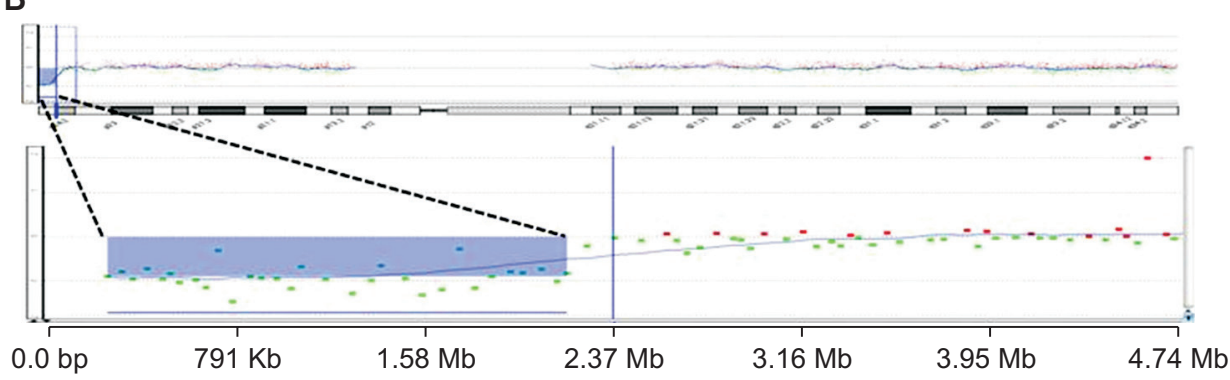

Fig. 2. Comparative genomic hybridization analysis showed 46, XY,duplication of 7q36.1-q36.3 (A), and deletion of 9p24.3 (B). 
showed normal development.

When he visited the clinic, he could walk upstairs, but he couldn't tiptoe or jump. In pediatric evaluation of disability inventory, the normative standard scores of self-care, mobility, and social function were 15.0, 24.4, and 14.5, respectively. In addition, he received a score of 38.65 in the social quotient. All of them were below 2 standard deviations. In the Bayley Scale of Infant and Toddler Development 3rd edition, the age equivalents for gross motor, fine motor, and cognition were 31,27 , and 27 months, respectively. The age equivalents for receptive and expressive language were 24 and 18 months, respectively, according to the sequenced language scale for infants. The results of the receptive and vocabulary test were below the 10th percentile, and the score for the Korean oral syntax expression comprehension test was below the 1 st percentile. He could imitate only 1 syllable. The patient had microcephaly and mild facial dysmorphic features, specifically a long face with high forehead, as well as a small nose and small ears (Fig. 1). On physical examination, deep tendon reflexes were normal, and no upper motor neuron signs were detected. Brain magnetic resonance imaging (MRI) indicated no definite abnormalities.

Laboratory studies did not reveal any specific findings, such as Wilson's disease, autoimmune disease, endocrine disease, or metabolic disease. Chromosome analysis and array comparative genomic hybridization (CGH) analysis showed 46,XY,dup(7)(q36.1-q36.3) and $\operatorname{del}(9)$ (p24.3) (Fig. 2). The duplication on chromosome 7, spanning 149,128,443-159,088,636 bp, was estimated to be 9.9 $\mathrm{Mb}$ in size, containing 68 known genes, while the deletion of chromosome 9, spanning 271,257-2,183,334 bp, was calculated to be $1.9 \mathrm{Mb}$ in size and included 6 known genes (Table 1). The genetic information is described on the following websites: Genome Browser (http://genome. ucsc.edu/cgi-bin/hgGateway), Online Mendelian Inheritance in Man (OMIM, http://www.ncbi.nlm.nih.gov/ omim) [1], DECIPHER (http://decipher.sanger.ac.uk) [2], and Ensembl genome browser (http://www.ensembl. org/homo_sapiens/location). Chromosome analysis and array CGH analysis of both parents were performed, and no specific findings were observed.

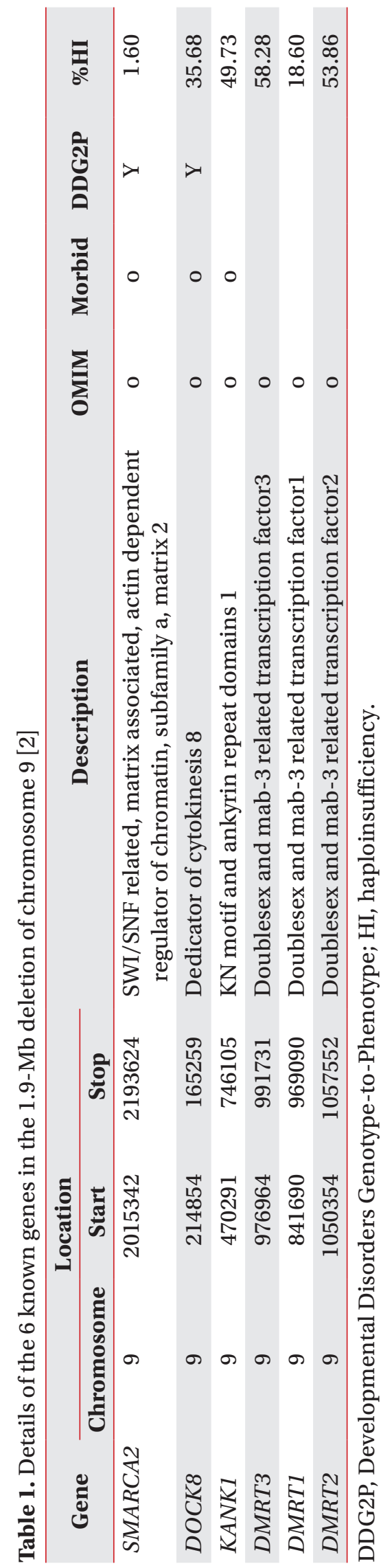

www.e-arm.org 


\section{DISCUSSION}

Global developmental delay and intellectual disability are relatively common pediatric conditions, affecting $3 \%$ of the general population. Furthermore, up to $40 \%$ of such developmental disability cases are caused by genetic factors. The American and European guidelines on this group state that genetic testing is recommended as a standardized diagnostic practice [3]. In addition, Miller et al. [4] recommend chromosomal microarray as a first-tier clinical diagnostic test for individuals with developmental disability. Establishing the cause of a child's disability can predict prognosis and other possible complications. Furthermore, it can assist in counselling parents and providing choices for therapeutic and educational interventions, as well as rehabilitation. Therefore, genetic study, including array CGH should be recommended for unexplained developmental delay or intellectual disability such as the case presented in this study.

Phenotypes of a few patients with the 7q36.1-q36.3 duplication or 9p24.3 deletion have been reported. However, to our knowledge, this is the first report of a patient harboring both copy number variations. Therefore, the genotype-phenotype correlation has not been clearly de- fined. The findings in our patient were compared to those of previously reported cases.

The $9 p$ deletion syndrome was first described in 1973. It is characterized by multiorgan syndrome, which includes dysmorphic facial features (e.g., trigonocephaly, midface hypoplasia, and long philtrum), hyperlaxity with frequent abdominal hernia, abnormalities of the extremities, and variable degrees of cognitive delay [5]. The deletion breakpoint-heterogeneous and variable in size-occurs from 9p22 to 9p24. In the present patient, the size of the deletion in chromosome 9 was $1.9 \mathrm{Mb}$. The 6 known genes in the 1.9-Mb deletion of chromosome 9 were described in Table 1. Some of the patient's characteristics were associated with $9 p$ deletion syndrome, e.g., midface hypoplasia, long philtrum, and intellectual disability.

Deletion of the short arm of chromosome 9 is also associated with sexual development disorders, and the region was recently further narrowed down to 9p24.3-pter, containing the doublesex- and mab-3-related transcription factor (DMRT) domain. DMRT genes are the strongest candidates for the gonadal dysgenesis phenotype, however the underlying molecular mechanism remains unclear [6]. A patient with complete $46, \mathrm{XY}$ gonadal dysgenesis and motor developmental retardation underwent

Table 2. Clinical manifestations associated with the duplication of $7 \mathrm{q} 36$ to the chromosome terminus

\begin{tabular}{llll}
\hline & \multicolumn{1}{c}{ Present study } & Boccone et al. [9] & Verma et al. [10] \\
\hline Duplication segment & dup(q36.1-q36.3) & dup(p36>qter) & dup(p36>qter) \\
Size (Mb) & 9.9 & Unknown & Unknown \\
\hline Chromosome position & $149,128,443-159,088,636$ & Unknown & Unknown \\
\hline Sex & Male & Female & Male \\
Age (yr) & 6 & 7 & 3.5 \\
Macrocephaly & No & Yes & No \\
Microcephaly & Yes & No & Yes \\
Frontal bossing & No & Yes & Yes \\
Developmental delay & Yes & Yes & Yes \\
Language developmental delay & Prominent & Prominent & Prominent \\
Electroencephalography & Not tested & Abnormal & Unknown \\
Brain imaging & Normal & Double cortex & Unknown \\
& & Arachnoidal & \\
Other findings & fronto-parietal cyst & \\
& Small nose and ears & Small nose & Short attention span \\
& High forehead & Joint hypermobility & Poor socialization \\
& Long face & Short 5th finger & Slightly increased tone \\
& & (clinodactyly) & Ankle clonus \\
& & & Macrocrania \\
\hline
\end{tabular}


a gonadectomy at 3.2 years of age [7], and histological analysis disclosed dysgenetic gonads with gonadoblastoma. The present patient shows normal external genitalia, and endocrine laboratory studies, including sex hormone analysis, showed findings within the normal range for his age.

Another gene in the deletion site of chromosome 9, dedicator of cytokinesis 8 (DOCK8, OMIM 611432), is expressed in the immune system, and mutation in this gene results in immune-related disorders [1]. Engelhardt et al. [8] reported the clinical phenotype of 64 patients with DOCK8 deficiency, and uncovered an association with frequent infection and high mortality at a young age. It is therefore noteworthy that the present patient had been hospitalized several times until 3 years due to recurrent episodes of pneumonia. Immunological evaluation was performed at our clinic, but the results were normal.

In the present patient, the duplication of chromosome 7 was estimated to be $9.9 \mathrm{Mb}$ in size, and it contained 68 genes, including 3 genes from the DDG2P, namely motor neuron and pancreas homeobox 1 (MNX1), sonic hedgehog $(\mathrm{SHH})$, and WD repeat-containing protein 60 (WDR60). Two patients with duplication from 7q36 to the chromosome terminus have been reported $[9,10]$, and both children showed developmental delay, significantly delayed speech and language, as well as frontal bossing (Table 2). Our patient had severe language and speech delay, however brain MRI showed no significant findings.

$M N X 1, S H H$, and WDR60 are in the DDG2P database because variants in these genes are associated with specific developmental phenotypes. MNX1 (OMIM 142994) encodes a transcription factor containing a homeobox domain. Loss-of-function mutations in MNX1 are associated with Currarino syndrome, which is characterized by anorectal, sacral, and presacral anomalies [1]. SHH (OMIM 600725) encodes a protein implicated in the patterning of the ventral neural tube, anterior-posterior limb axis, and ventral somites in the early embryo. $\mathrm{SHH}$ mutations have been found to underlie the following comorbidities: holoprosencephaly-3, single median maxillary central incisor, schizencephaly, and microphthalmia with coloboma-5 [1]. WDR60 (OMIM 615462) encodes a member of the WD repeat protein family, which is involved in a variety of cellular processes such as cell cycle progression, signal transduction, and apoptosis. Mutations in this gene have been found to be related to short- rib polydactyly and Jeune syndrome [1].

The work presented here reports the first case of concurrent de novo duplication of 7q36.1-q36.3 and deletion of 9 p24.3. Although some characteristics were similar to those of previously reported cases, no specific genotypephenotype correlations were established. Further cumulative data based on the molecular approach are warranted to understand the role and influence of the genes in the 7q36.1-q36.3 duplication and 9p24.3 deletion regions. Additionally, if a national registration system for sharing genetic information had existed in Korea, multi-center studies with larger subjects would have been easily possible, and this concept could prove beneficial for future studies.

\section{CONFLICT OF INTEREST}

No potential conflict of interest relevant to this article was reported.

\section{ACKNOWLEDGMENTS}

This study was supported by the Research Fund of Seoul St. Mary's Hospital, The Catholic University of Korea.

\section{REFERENCES}

1. Online Mendelian Inheritance in Man (OMIM) [Internet]. Bethesda, MD: National Center for Biotechnology Information, US National Library of Medicine; c2017 [cited 2017 Sep 1]. Available from: https://www. ncbi.nlm.nih.gov/omim.

2. DECIPHER (DatabasE of genomiC varIation and Phenotype in Humans using Ensembl Resources). GRCh37, mapping the clinical genome [Internet]. Cambridge: Wellcome Trust Sanger Institute; c2017 [cited 2017 Sep 1]. Available from: https://decipher. sanger.ac.uk/.

3. Miclea D, Peca L, Cuzmici Z, Pop IV. Genetic testing in patients with global developmental delay / intellectual disabilities: a review. Clujul Med 2015;88:288-92.

4. Miller DT, Adam MP, Aradhya S, Biesecker LG, Brothman AR, Carter NP, et al. Consensus statement: chromosomal microarray is a first-tier clinical diagnostic test for individuals with developmental disabilities or congenital anomalies. Am J Hum Genet 2010;86:749- 
64.

5. Spazzapan P, Arnaud E, Baujat G, Nizon M, Malan V, Brunelle F, et al. Clinical and neuroradiological features of the $9 p$ deletion syndrome. Childs Nerv Syst 2016;32:327-35.

6. Barbaro M, Balsamo A, Anderlid BM, Myhre AG, Gennari $\mathrm{M}$, Nicoletti A, et al. Characterization of deletions at $9 p$ affecting the candidate regions for sex reversal and deletion 9p syndrome by MLPA. Eur J Hum Genet 2009;17:1439-47.

7. Del Rey G, Venara M, Papendieck P, Gruneiro L, Tangari A, Boywitt A, et al. Association of distal deletion of the short arm of chromosome 9 with 46,XY disorder of sex development and gonadoblastoma. Biol Syst Open Access 2015;4:129.

8. Engelhardt KR, Gertz ME, Keles S, Schaffer AA, Sigmund EC, Glocker $\mathrm{C}$, et al. The extended clinical phenotype of 64 patients with dedicator of cytokinesis 8 deficiency. J Allergy Clin Immunol 2015;136:402-12.

9. Boccone L, Gasperini D, Pilloni G, Cao A, Nucaro A. Duplication of the terminal band of the long arm of chromosome 7: a new case. Genet Couns 2004;15:8790.

10. Verma RS, Conte RA, Pitter JH. Tandem duplication of the terminal band of the long arm of chromosome 7 (dir dup (7)(q36-qter)). J Med Genet 1992;29:344-5. 